\title{
Psicooncología
}

ISSN: $1696-7240$

\section{Afrontamiento y calidad de vida en pacientes con cáncer de mama: Es- tudio preliminar de semejanzas y diferencias entre pacientes con y sin metástasis}

\author{
Antoni Font Guiteras ${ }^{1 *}$; Paula Reventós Osuna ${ }^{2}$; Catalina Falo Zamora ${ }^{3}$
}

Recibido el 1 de septiembre de 2019 / Aceptado: 24 de febrero de 2020.

Resumen: Objetivo: Obtener información sobre las estrategias de afrontamiento que utilizan las pacientes de cáncer de mama y su relación con la calidad de vida, diferenciando dos situaciones de salud: pacientes con y sin metástasis, atendidas en un mismo hospital oncológico especializado de nuestro entorno. Método: 60 mujeres con cáncer de mama fueron entrevistadas individualmente y completaron dos instrumentos que medían diversos componentes de afectación de la calidad de vida (QLTF) y cinco maneras diferentes de enfrentarse a la enfermedad (test mini-MAC). Resultados: Las estrategias de afrontamiento se relacionan con la calidad de vida de las pacientes con cáncer de mama. En nuestro estudio observamos que responder a la situación oncológica mediante "Preocupación Ansiosa" o Desesperanza" correlaciona con una mayor afectación de la calidad de vida general. Sin embargo, no se observan diferencias significativas entre pacientes con metástasis y sin metástasis en cuanto a las estrategias utilizadas. Las pacientes con metástasis presentan más dificultades en la realización de sus actividades cotidianas, pero el malestar emocional es similar en ambos grupos de pacientes.

Conclusión: En congruencia con estudios previos, las estrategias y respuestas de afrontamiento activo se relacionan con mejor calidad de vida. Aunque las pacientes con metástasis presentan más dificultades y afectación de la calidad de vida, parece ser que se enfrentan a la enfermedad de manera similar a las pacientes sin metástasis.

Palabras clave: Cáncer de mama; estrategias de afrontamiento; calidad de vida; metástasis.

[en] Coping and quality of life in patients with breast cancer: Preliminary study about similarities and differences between patients with and without metastasis.

1 Antoni Font Guiteras. Unitat de Psicologia Bàsica. Facultat de Psicologia

Universitat Autònoma de Barcelona

E-mail: Antonio.Font@uab.cat

2 Paula Reventós Osuna Unitat de Psicologia Bàsica. Facultat de Psicologia

Universitat Autònoma de Barcelona

E-mail: paula.reventos@e-campus.uab.cat

3 Catalina Falo Zamora Institut Català d'Oncologia. L'Hospitalet de Llobregat, Barcelona.

E-mail: cfalo@iconcologia.net

* Dirección de correspondencia: Prof. Dr. Antoni Font Guiteras. Grup d’Investigació en Estrès i Salut (GIES) / Stress and Health Research Group / Grupo de Investigación en Estrés y Salud Unitat de Psicologia Bàsica. Facultat de Psicologia · Carrer de la Fortuna s/n. Campus de la Universitat Autònoma de Barcelona 08193 Bellaterra (Cerdanyola del Vallès) · Barcelona · España. E-mail: Antonio.Font@uab.cat 


\begin{abstract}
Objective: To obtain information about the coping strategies used by breast cancer patients, and their relationship with quality of life, differentiating two health situations: patients with and without metastasis, treated in the same specialized hospital of our environment. Method: 60 women with breast cancer were interviewed individually and completed two instruments that measured various components of quality of life (QLTF) and five different ways of coping with the disease (mini-MAC test). Results: Coping strategies are related to the quality of life of patients with breast cancer. In our study, we observed that responding to the oncological situation by means of "Anxious concerns" or "Hopelessness" correlates with a greater affectation of the general quality of life. However, no significant differences are observed between patients with metastasis and without metastasis regarding the strategies. Patients with metastasis present more difficulties in carrying out their daily activities, but the emotional discomfort is similar in both groups. Conclusion: In congruence with previous studies, the active coping strategies and responses are related to a better quality of life. Although patients with metastases show more difficulties and quality of life impairments, it seems that they face the disease in a similar way to patients without metastases.
\end{abstract}

Keywords: Breast cancer; coping strategies; quality of life,; metastasis.

Sumario: 1. Introducción 2. Método 3. Resultados 4. Discusión 5. Conclusión. 6 Referencias bibliográficas

Como citar: Font Guiteras A, Reventós Osuna P, Falo Zamora, CD. Afrontamiento y calidad de vida en pacientes con cáncer de mama: Estudio preliminar de semejanzas y diferencias entre pacientes con y sin metástasis. Psicooncología 2020;17:179-194. doi: 10.5209/psic.68249.

\title{
1. Introducción
}

El cáncer es una de las principales causas de mortalidad prematura a nivel mundial. El cáncer de mama, representa el $16 \%$ de todos los cánceres femeninos ${ }^{(1)}$. Tanto la enfermedad como su tratamiento pueden afectar a la calidad de vida de las pacientes y exigen importantes esfuerzos de adaptación a lo largo de todo el proceso oncológico ${ }^{(2-4)}$. En la valoración de gravedad de un suceso intervienen las herramientas de las que dispone cada persona para enfrentarse y dar respuesta a la situación, tal como ya indicaron Lazarus y Folkman ${ }^{(5)}$ al definir el afrontamiento como los esfuerzos cognitivos y/o conductuales para intentar reducir las situaciones que se valoran como estresantes y que ponen a prueba los recursos del sujeto. Cuando el cáncer ya está presente, las personas tienen poco control objetivo sobre los acontecimientos que siguen al diagnóstico, por lo que las estrategias de afrontamiento se centran en mantener el equilibrio emocional o disminuir la ansiedad generada por las situaciones oncológicas y su valoración, en las diferentes fases de la enfermedad, como el miedo a la recidiva en la fase de intervalo libre, o los efectos secundarios del tratamiento de las metástasis mediante quimioterapia en estadios avanzados ${ }^{(6-8)}$.

Watson y $\mathrm{Greer}^{(9)}$ propusieron que los pacientes, ante el diagnóstico, podrían generar cinco respuestas o estilos de afrontamiento diferentes: espíritu de lucha, negación, fatalismo, desesperanza o preocupación ansiosa. Estas reacciones influirían en la valoración subjetiva del diagnóstico, la percepción de control y el nivel de adaptación. Si el diagnóstico de cáncer se percibe como una gran amenaza, la persona podrá desarrollar diferentes respuestas como la negación, el fatalismo o la preocupación ansiosa. En cambio, cuando es percibido como un desafío, la persona entiende que existe un cierto control de la situación y el espíritu de lucha será más probable como 
estrategia de afrontamiento ${ }^{(10,11)}$. Haber ${ }^{(12)}$ concluye que el mejor indicador para saber cómo sobrellevará el proceso de enfermedad una mujer afectada de cáncer de mama, será analizando su modo de confrontar situaciones traumáticas. El afrontamiento activo a la enfermedad grave se asocia a una reducción de la tensión producida por la patología, en cambio, en las pacientes que tienen una disposición más pasiva, se observa un mayor deterioro de la calidad de $\operatorname{vida}^{(13,14)}$. Además, la pasividad y la preocupación ansiosa se han asociado no sólo a un aumento en la tensión emocional, sino que algunos estudios sugieren también un progreso más rápido de la enfermedad ${ }^{(15,16)}$. En este mismo sentido, Dunkel-Schetter y colaboradores ${ }^{(17)}$ constataron que los estilos de afrontamiento pasivos como el fatalismo, la preocupación ansiosa y la desesperanza también se mostraron asociados a un peor pronóstico en la evolución de la enfermedad y a un mayor malestar psicológico. En el estudio de Mytko et al. ${ }^{(18)}$ evaluaron a 49 pacientes de cáncer con diferentes diagnósticos que iban a someterse a altas dosis de quimioterapia, observándose una correlación significativa entre el modo de afrontamiento de negación-evitación y el malestar psicológico. En una muestra de 101 mujeres diagnosticadas de cáncer avanzado, las respuestas de afrontamiento "espíritu de lucha" y "expresión emocional" se asociaron a un mejor ajuste a la enfermedad que las respuestas "fatalismo" y "negación"(19). Hack y Degner ${ }^{(20)}$ observaron que las mujeres con bajo afrontamiento evitativo conseguían un mejor ajuste y se involucraban de una forma más activa en la toma de decisiones sobre su tratamiento. Por otra parte, en el estudio de Mera y Ortiz ${ }^{(21)}$, se constató una relación significativa entre el optimismo y la utilización de estrategias de afrontamiento activo. También se ha comparado los estilos de afrontamiento al cáncer de mama de las pacientes con y sin depresión asociada a la enfermedad, observándose que entre las pacientes que tenían depresión predominaba el uso de las estrategias "desesperanza", "preocupación ansiosa" y "fatalismo", mientras que las pacientes sin depresión hacían un mayor uso de la estrategia de afrontamiento denominada "espíritu de lucha"(22). Torrecilla et al. ${ }^{(23)}$ 1levaron a cabo un estudio para evaluar las estrategias de afrontamiento y calidad de vida en 80 pacientes oncológicas. Los resultados mostraron que las estrategias más utilizadas por las mujeres del estudio eran las pasivas o de evitación del problema, específicamente la evitación cognitiva (pasiva cognitiva) y la estrategia de descarga emocional (pasiva conductual). La estrategia de afrontamiento "evitación" correlacionó negativamente con la percepción de calidad de vida.

Así pues, los diversos trabajos revisados constatan que la calidad de vida de las pacientes con cáncer de mama depende en parte de las respuestas de afrontamiento que utilizan a lo largo del proceso de la enfermedad. No obstante, se observan también algunas controversias en relación a las condiciones concretas en que se darían estas relaciones como la gravedad de la situación médica, o si determinadas estrategias como la negación puede resultar o no beneficiosas para algunas pacientes en determinadas circunstancias. En el estudio pionero de Greer et al. ${ }^{(24)}$, se observó que dicha estrategia correlacionaba con una mayor supervivencia en pacientes con cáncer de mama. En cambio, en estudios más recientes como por ejemplo el de Hack y Degner ${ }^{(20)}$ y Mera y Ortiz ${ }^{(21)}$ dicha estrategia correlacionaría desfavorablemente con la calidad de vida y la implicación de la paciente en el tratamiento, lo que disminuiría las posibilidades de controlar la enfermedad. Además, subsiste el problema de diferenciar las estrategias dado que no son necesariamente excluyentes y que las mismas pacientes pueden utilizar estrategias contrarias, según el momento o la situación ${ }^{(25)}$. Recientemente se han propuesto diversos niveles de análisis para diferenciarlas, pero los problemas 
conceptuales persisten $^{(26)}$. Por otra parte, diferentes estrategias podrían asociarse a mayor o menor efectividad para mantener la calidad de vida en función del momento de la enfermedad o la gravedad de la situación oncológica, como la presencia o no de metástasis. Butler et al. ${ }^{(27)}$ corroboró que las pacientes diagnosticadas de cáncer con metástasis, presentan síntomas físicos y emocionales importantes y cuando sus expectativas empeoran con el tiempo, se observa una disminución progresiva de la calidad de vida. En la investigación de Hagedorn et al. ${ }^{(28)}$, las pacientes diagnosticadas de cáncer sin metástasis indicaron frecuentemente tener miedo sobre el futuro, pero éste disminuía con el tiempo mientras se mantenía un pronóstico favorable. En el trabajo de Wang et al. ${ }^{(29)}$ se observó que el espíritu de lucha favorecía la adaptación al cáncer de mama, pero únicamente en la fase de diagnóstico. Pastells y Font ${ }^{(30)}$ constataron que el pesimismo se relacionaba significativamente con la pérdida de calidad de vida de las pacientes con cáncer de mama independientemente de si recibían o no tratamiento en el momento de la valoración. Como indican Cheng et al. ${ }^{(31)}$, la relación entre el afrontamiento al cáncer y la adaptación al mismo no está totalmente clarificada, no sólo por dificultades conceptuales, sino también por la diversidad de situaciones de salud consideradas en los estudios.

El presente trabajo tiene como objetivo ampliar la información sobre las estrategias de afrontamiento que utilizan las pacientes de cáncer de mama que son tratadas en un mismo entorno sanitario y comparar la calidad de vida y las respuestas de las pacientes con y sin metástasis.

Planteamos las siguientes hipótesis:

1. La calidad de vida será más favorable en el grupo de pacientes sin metástasis (las pacientes con metástasis presentarán más síntomas físicos y emocionales).

2. Dado que las respuestas a la enfermedad dependen en gran medida de factores personales, ambos grupos de pacientes utilizarán formas de similares de afrontamiento.

3. Las respuestas de afrontamiento se diferenciarán respecto a su eficacia para preservar la calidad de vida.

\section{Método}

\section{Muestra}

Para realizar esta investigación se dispuso de una muestra de sesenta mujeres diagnosticadas de cáncer de mama, la mitad de ellas con metástasis en diferentes localizaciones, mayoritariamente metástasis ósea. Este estudio forma parte de una investigación longitudinal más amplia que se está realizando en el Instituto Catalán de Oncología (ICO-Hospitalet). La muestra procede de pacientes atendidas en el Servicio de Oncología Médica del Hospital Universitario de Bellvitge. de A modo de estudio preliminar, se han analizado las respuestas de afrontamiento de las 30 primeras pacientes de la muestra que presentaban metástasis y las 30 primeras sin metástasis. Los criterios de inclusión fueron tener diagnóstico de cáncer de mama y ser tratada en el mencionado hospital. El único criterio de exclusión fue presentar dificultades cognitivas para entender las preguntas, lo que coincidió con las pacientes de más de 80 años (se excluyeron 7 pacientes). Las características socio-demográficas y clínicas se indican en la tabla 1. 


\section{Instrumentos}

Los instrumentos utilizados para dar respuesta a los objetivos del estudio son los siguientes:

Test Mini-MAC, compuesto por 29 ítems, que evalúa las respuestas cognitivas y comportamentales al cáncer, en escalas de Likert de 4 puntos. Permite identificar las siguientes cinco formas de afrontamiento, en la versión española utilizada, validada por Costa-Requena y $\mathrm{Gil}^{(32)}$ :

- Indefensión/desesperanza (ID): la persona tiene fuertes sentimientos de vulnerabilidad debido a la enfermedad y la percepción de control es prácticamente nula. Ante ello, acaba adquiriendo una actitud negativa y desesperanzada.

- Preocupación ansiosa (PA): la persona afectada percibe que la enfermedad pone en riesgo su vida y lo vive como una gran amenaza que le produce un sentimiento constante de preocupación y ansiedad frente a los sucesos futuros.

- Evitación/negación (EN): se produce un rechazo del diagnóstico o se minimiza la importancia de la enfermedad, evitando de esta forma pensar en ello.

- Espíritu de lucha (EL): la persona interpreta el diagnóstico de la enfermedad como un reto frente al cual considera que puede tener un cierto de control. Se da una respuesta activa de afrontamiento, involucrándose en actividades que puedan favorecer su adaptación a la enfermedad, con una actitud optimista sobre el pronóstico y con aceptación en el diagnóstico.

- Fatalismo/aceptación estoica (FAE): Se acepta el diagnóstico con resignación o actitud fatalista considerando que no se puede ejercer control alguno sobre el devenir de los acontecimientos.

En nuestro estudio obtuvimos un valor alfa de Cronbach de 0,74 para el conjunto de la escala.

Cuestionario de calidad de vida QL-CA-AFex ${ }^{(33)}$. Se trata de un instrumento autoadministrable para personas afectadas por cáncer que mide la calidad de vida percibida en relación con la salud, desarrollado en nuestro país y utilizado en varios estudios con pacientes de cáncer de $\mathrm{mama}^{(30,34,35)}$. Consta de 27 ítems (escalas visuales análogas) que se puntúan de 0 a 100 . Se agrupan en cuatro subescalas: síntomas (6 ítems), autonomía (5 ítems), dificultades familiares y sociales (8 ítems) y dificultades psicológicas ( 7 ítems). El último ítem es una escala lineal global referida a la valoración general de la calidad de vida de la última semana. En todos los casos, una mayor puntuación hace referencia a un mayor impacto de la enfermedad, es decir una mayor pérdida de calidad de vida. En la muestra del estudio se obtuvo un valor de fiabilidad alfa de Cronbach para toda la escala, de 0,85.

\section{Procedimiento}

El presente estudio se ha realizado con la aprobación del comité de ética del Hospital Universitario de Bellvitge (HUB). La información perteneciente a los pacientes integrantes del estudio se ha tratado de acuerdo a lo establecido en la Ley Orgánica 15/1999 de protección de datos personales.

Las pacientes fueron entrevistadas individualmente en el Instituto Catalán de Oncología. Previamente se solicitó el consentimiento informado para participar en la 
investigación. Los oncólogos derivaron a las pacientes a una psicóloga que realizaba la entrevista en el hospital, el mismo día de la visita médica. Posteriormente a la presentación del proyecto y explicación de procedimiento de recogida de datos, las pacientes completaban los cuestionarios en presencia de la psicóloga a la que podían preguntar en caso de duda. Al formar parte de una investigación más amplia se aplicaron diversas pruebas a lo largo de aproximadamente 60 minutos. Los datos del presente estudio se circunscriben a las respuestas reportadas por las primeras pacientes evaluadas, en los instrumentos Mini-MAC y QL-CA-AFex.

\section{Análisis de datos}

Se han realizado pruebas de comparación de medias con muestras independientes no paramétricas Mann Whitney y análisis de correlaciones Rho Spearman mediante el programa estadístico SPSS versión 21.0. El nivel de significación mínimo se ha fijado en $\mathrm{p}<0,05$.

\section{Resultados}

La media de edad es de 58 años (rango entre 36 y 84 años) y la desviación típica es de 10,58. El 85\% de la muestra tiene estudios primarios o medios, el 58,3\% están casadas, un 58,3\% tiene antecedentes familiares de cáncer y un 53,3\% no trabaja por motivos de salud. Los datos clínicos indican que un $93,3 \%$ han sido intervenidas quirúrgicamente y un $56,7 \%$ hace uso de psicofármacos. Entre los dos grupos (pacientes sin y con metástasis) no hay diferencias significativas respecto a las características demográficas y médicas, excepto en la variable "cirugía": las cuatro participantes no intervenidas quirúrgicamente pertenecen al grupo de las pacientes con metástasis (ver tabla 1).

Tabla 1. Características socio-demográficas y médicas de la muestra.

Valores P de la prueba Chi-cuadrado de comparación de proporciones

\begin{tabular}{|c|c|c|c|c|c|}
\hline Variables & Categorías & $\mathrm{N}=60(\%)$ & $\begin{array}{l}\mathrm{N}=30 \text { Pacientes } \\
\text { con metástasis }\end{array}$ & $\begin{array}{c}\mathrm{N}=\mathbf{3 0} \\
\text { Pacientes sin } \\
\text { metástasis }\end{array}$ & $\begin{array}{l}\text { Valor } \\
\text { de } P \text {. }\end{array}$ \\
\hline \multirow{4}{*}{ Edad } & De 36 a 46 años & $7(11,7 \%)$ & $2(28,6 \%)$ & $5(71,4 \%)$ & \multirow{4}{*}{0,76} \\
\hline & De 47 a 57 años & $\begin{array}{c}23 \\
(38,3 \%) \\
\end{array}$ & $9(39,1 \%)$ & $14(60,9 \%)$ & \\
\hline & De 58 a 68 años & $\begin{array}{c}17 \\
(28,3 \%)\end{array}$ & $11(64,7 \%)$ & $6(35,3 \%)$ & \\
\hline & De 69 a 84 años & $\begin{array}{c}13 \\
(21,7 \%)\end{array}$ & $8(61,5 \%)$ & $5(38,5 \%)$ & \\
\hline & & & $\frac{\text { Media de edad: }}{\underline{60,8}}$ & $\begin{array}{l}\text { Media de } \\
\text { edad: } 55,7 \\
\end{array}$ & \\
\hline \multirow{2}{*}{ Metástasis } & Sí & $30(50 \%)$ & $30(50 \%)$ & $30(50 \%)$ & \multirow[b]{2}{*}{-} \\
\hline & No & $30(50 \%)$ & $30(50 \%)$ & $30(50 \%)$ & \\
\hline
\end{tabular}




\begin{tabular}{|c|c|c|c|c|c|}
\hline Variables & Categorías & $\mathrm{N}=60(\%)$ & $\begin{array}{l}\mathrm{N}=30 \text { Pacientes } \\
\text { con metástasis }\end{array}$ & $\begin{array}{c}\mathrm{N}=\mathbf{3 0} \\
\text { Pacientes sin } \\
\text { metástasis }\end{array}$ & $\begin{array}{l}\text { Valor } \\
\text { de } P \text {. }\end{array}$ \\
\hline \multirow[t]{2}{*}{ Cirugía } & Sí & $\begin{array}{c}56 \\
(93,3 \%)\end{array}$ & $26(46,4 \%)$ & $30(53,6 \%)$ & \multirow[t]{2}{*}{$0,04 *$} \\
\hline & No & $4(6,7 \%)$ & $4(100 \%)$ & 0 & \\
\hline \multirow{5}{*}{ Estado civil } & Casada & $\begin{array}{c}35 \\
(58,3 \%) \\
\end{array}$ & $17(48,6 \%)$ & $18(51,4 \%)$ & \multirow{5}{*}{0,528} \\
\hline & Soltera & $7(11,7 \%)$ & $3(42,9 \%)$ & $4(57,1 \%)$ & \\
\hline & Pareja estable & $3(5 \%)$ & $0(0 \%)$ & $3(100 \%)$ & \\
\hline & Divorciada & $12(20 \%)$ & $8(66,7 \%)$ & $4(33,3 \%)$ & \\
\hline & Viuda & $3(5 \%)$ & $2(66,7 \%)$ & $1(33,3 \%)$ & \\
\hline \multirow{3}{*}{ Estudios } & Primarios & $\begin{array}{c}26 \\
(43,3 \%)\end{array}$ & $14(53,8 \%)$ & $12(46,2 \%)$ & \multirow{3}{*}{0,584} \\
\hline & Medios & $\begin{array}{c}25 \\
(41,7 \%) \\
\end{array}$ & $12(48 \%)$ & $13(52 \%)$ & \\
\hline & Superiores & $9(15 \%)$ & $4(44,4 \%)$ & $5(55,6 \%)$ & \\
\hline \multirow{2}{*}{$\begin{array}{c}\text { Antecedentes } \\
\text { familiares }\end{array}$} & Sí & $\begin{array}{c}38 \\
(63,3 \%)\end{array}$ & $20(52,6 \%)$ & $18(47,4 \%)$ & \multirow{2}{*}{0,194} \\
\hline & No & $\begin{array}{c}22 \\
(36,7 \%)\end{array}$ & $10(45,5 \%)$ & $12(54,5 \%)$ & \\
\hline \multirow{2}{*}{$\begin{array}{c}\text { Uso } \\
\text { psicofármacos }\end{array}$} & Sí & $\begin{array}{c}34 \\
(56,7 \%) \\
\end{array}$ & $15(44,1 \%)$ & $19(55,9 \%)$ & \multirow{2}{*}{0,595} \\
\hline & No & $\begin{array}{c}26 \\
(43,3 \%)\end{array}$ & $15(57,7 \%)$ & $11(42,3 \%)$ & \\
\hline \multirow{3}{*}{$\begin{array}{l}\text { Situación } \\
\text { laboral }\end{array}$} & Trabaja & \begin{tabular}{|l|}
$8(13,3 \%)$ \\
\end{tabular} & $0(0 \%)$ & $8(100 \%)$ & \multirow{3}{*}{0,606} \\
\hline & $\begin{array}{c}\text { No trabaja por } \\
\text { motivos de } \\
\text { salud }\end{array}$ & $\begin{array}{c}32 \\
(53,3 \%)\end{array}$ & $17(53,1 \%)$ & $15(46,9 \%)$ & \\
\hline & $\begin{array}{l}\text { No trabaja por } \\
\text { otros motivos }\end{array}$ & $\begin{array}{c}19 \\
(31,7 \%)\end{array}$ & $13(68,4 \%)$ & $6(31,6 \%)$ & \\
\hline
\end{tabular}


Tabla 2. Medias y significatividad de la afectación en los ítems, las escalas y la valoración general de la calidad de vida. prueba $U$ de Mann Whitney, significativa al nivel de $\mathrm{p}<0,05$.

\begin{tabular}{|c|c|c|c|}
\hline Variables $(N=60)$ & $\begin{array}{c}\text { Media } \\
\text { Grupo sin } \\
\text { metástasis } \\
\end{array}$ & $\begin{array}{c}\text { Media } \\
\text { Grupo con } \\
\text { metástasis }\end{array}$ & $\begin{array}{l}\text { Valor } \\
\text { de } P\end{array}$ \\
\hline ESCALA DE SÍNTOMAS & 23,26 & 23,70 & 0,912 \\
\hline Náuseas & 27,75 & 29,25 & 0,606 \\
\hline Dolor & 25,75 & 27,14 & 0,734 \\
\hline Fatiga & 24,85 & 27,91 & 0,463 \\
\hline Dificultades para estar despierto & 24,65 & 27,20 & 0,519 \\
\hline Dificultades para dormir & 26,42 & 26,57 & 0,970 \\
\hline Apetito & 26,61 & 29,34 & 0,475 \\
\hline $\begin{array}{c}\text { ESCALA DE DIFICULTADES EN LOS } \\
\text { HÁBITOS COTIDIANOS }\end{array}$ & 20,92 & 29,73 & $0,029(*)$ \\
\hline Dificultad movilidad & 26,39 & 31,52 & 0,171 \\
\hline $\begin{array}{l}\text { Dificultades en las actividades de cuidado } \\
\text { personal }\end{array}$ & 28,07 & 31,02 & 0,394 \\
\hline Dificultades en actividades físicas & 22,89 & 31,27 & $0,034(*)$ \\
\hline Dificultades en actividades de casa & 20,62 & 31,17 & $0,009(*)$ \\
\hline $\begin{array}{l}\text { ESCALA DE LAS DIFICULTADES } \\
\text { FAMILIARES Y SOCIALES }\end{array}$ & 20,96 & 19,88 & 0,773 \\
\hline Insatisfacción social & 24,42 & 29,48 & 0,176 \\
\hline Preocupación por los hijos & 24,44 & 30,14 & 0,164 \\
\hline Dificultades relación de pareja & 22,42 & 20,28 & 0,560 \\
\hline Dificultades relaciones familiares & 25,21 & 29,63 & 0,240 \\
\hline Pérdida ilusión & 27,44 & 29,48 & 0,614 \\
\hline Dificultades económicas & 27,20 & 29,80 & 0,515 \\
\hline ESCALA DE MALESTAR EMOCIONAL & 21,46 & 26,44 & 0,212 \\
\hline $\begin{array}{l}\text { Percepción de pérdida de atractivo para el } \\
\text { otro sexo }\end{array}$ & 27,69 & 28,28 & 0,881 \\
\hline Ira & 28,98 & 27,05 & 0,618 \\
\hline Ansiedad & 26,23 & 27,74 & 0,710 \\
\hline Insatisfacción con el aspecto físico & 21,52 & 30,31 & $0,026(*)$ \\
\hline Miedo & 24,92 & 27,96 & 0,448 \\
\hline Afectación estado de ánimo & 32,50 & 28,50 & 0,301 \\
\hline Dificultades concentración & 23,44 & 29,56 & 0,096 \\
\hline $\begin{array}{c}\text { AFECTACIÓN CALIDAD DE VIDA } \\
\text { GENERAL }\end{array}$ & 22,79 & 28 & 0,142 \\
\hline
\end{tabular}


En la tabla 2 se presentan los resultados de comparar la afectación de la calidad de vida entre ambos grupos de pacientes. Las pacientes con metástasis presentan mayor afectación en 22 de los 27 aspectos considerados, pero de manera significativa $(p<0,05)$ únicamente en cuatro: "dificultad para realizar actividades físicas", "dificultades para realizar actividades de casa" y en "insatisfacción con el aspecto físico"; y en la escala "dificultades en los hábitos cotidianos". Por su parte, las pacientes sin metástasis muestran mayor afectación en la escala "dificultades familiares y sociales", y en los ítems "dificultades en la relación de pareja", "ira" y "depresión", aunque las diferencias no son significativas.

Respecto a las estrategias utilizadas por ambos grupos de pacientes, la prueba de comparación U de Mann Whitney no aportó diferencias significativas (ver Tabla 3).

Tabla 3. Comparación de medias observadas en las estrategias de afrontamiento del MiniMAC (grupo con versus sin metástasis). Prueba de U de Mann Whitney.

\begin{tabular}{|l|c|c|c|}
\hline Estrategias de Afrontamiento & $\begin{array}{c}\text { Pacientes sin } \\
\text { metástasis } \\
\mathbf{( N = 3 0 )}\end{array}$ & $\begin{array}{c}\text { Pacientes con } \\
\text { metástasis } \\
\text { (N=30) }\end{array}$ & Valor de P. \\
\hline Espíritu de Lucha & 29,24 & 30,73 & 0,700 \\
\hline Desesperanza & 29,50 & 30,48 & 0,326 \\
\hline Preocupación Ansiosa & 28 & 31 & 0,231 \\
\hline Evitación Cognitiva & 28,62 & 31,33 & 0,440 \\
\hline Fatalismo & 31,69 & 28,37 & 0,359 \\
\hline
\end{tabular}

Por otra parte, para analizar la posible relación entre las respuestas de afrontamiento y la calidad de vida se calcularon las correlaciones Rho Spearman entre ambas escalas (tabla 4). La respuesta de afrontamiento denominada "Preocupación Ansiosa" se relacionó significativamente con una mayor disminución de la calidad de vida general, con mayor sensación de náuseas, con pérdida de ilusión por las cosas y con la escala de malestar emocional. La estrategia de "Evitación Cognitiva" se relacionó de forma significativa con mayor impacto en las escalas de afectación de los hábitos cotidianos y en Dificultades familiares y sociales, y con el ítem dificultades en el cuidado personal. La estrategia de afrontamiento denominada "Espíritu de Lucha" se relacionó significativamente con mayor afectación de los hábitos cotidianos y con el ítem de dificultades en el cuidado personal. Por otra parte, aunque no de manera significativa, el afrontamiento de "espíritu de lucha" correlacionó con menor presencia de dificultades familiares y sociales, y con menor malestar emocional. La "Desesperanza" solo se relacionó con la afectación de la Calidad de vida general. La respuesta denominada "Fatalismo" se relacionó significativamente con la fatiga, las dificultades en actividades físicas, las dificultades en actividades en casa, la preocupación por los hijos, y la pérdida de ilusión por las cosas. 


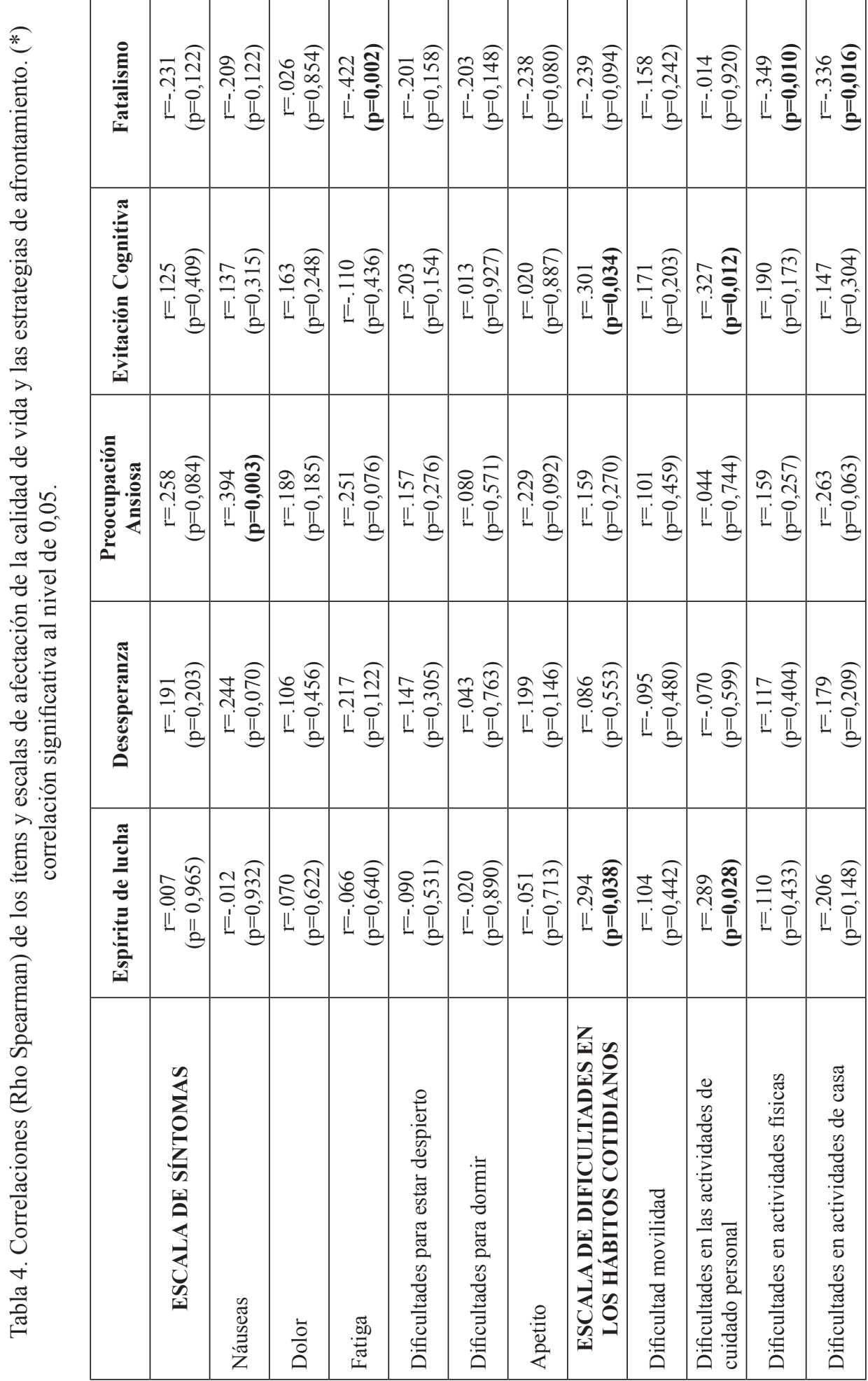




\begin{tabular}{|c|c|c|c|c|c|c|c|c|c|c|c|c|c|c|c|}
\hline 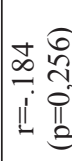 & 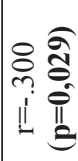 & 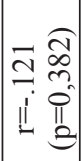 & {$\left[\begin{array}{cc}0 & \approx \\
\infty & 0 \\
0 & 0 \\
1 & 0 \\
1 & 0\end{array}\right.$} & 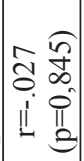 & 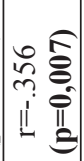 & $\mid \begin{array}{cc}2 & \widehat{3} \\
\infty & 0 \\
1 & 0 \\
1 & 0 \\
-1 & 3\end{array}$ & 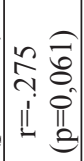 & 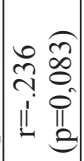 & 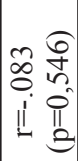 & 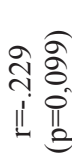 & 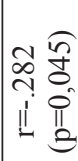 & 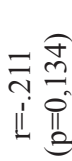 & 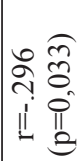 & 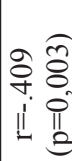 & 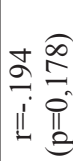 \\
\hline 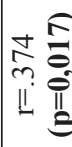 & 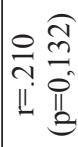 & 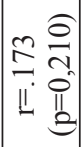 & 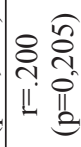 & 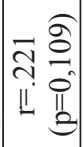 & 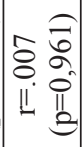 & 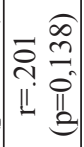 & $\left|\begin{array}{ll}0 & 0 \\
& \ddots \\
11 & 0 \\
11 & 0 \\
& 0\end{array}\right|$ & 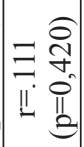 & $\begin{array}{ll}0 & \widehat{\sigma} \\
\hat{0} & 2 \\
i & 0 \\
1 & \|\end{array}$ & $\begin{array}{l}0 \\
0 \\
0 \\
0 \\
\| \\
\| \\
=\end{array}$ & 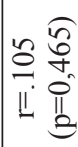 & 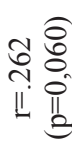 & 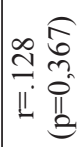 & 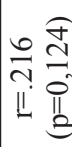 & 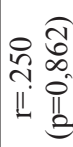 \\
\hline 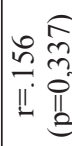 & 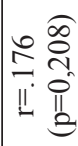 & $\begin{array}{ll}\infty & \widehat{n} \\
\infty & 0 \\
0 & n \\
0 & 0 \\
11 & 0 \\
1 & 0\end{array}$ & 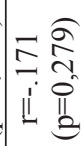 & 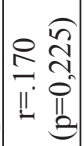 & 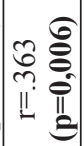 & 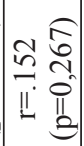 & $\left|\begin{array}{cc}0 & \widehat{a} \\
\infty & 0 \\
i & 0 \\
11 & 0 \\
1 & 0\end{array}\right|$ & 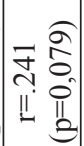 & 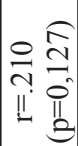 & 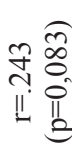 & 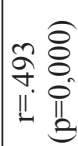 & 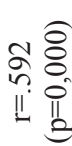 & $\begin{array}{ll}0 & 0 \\
0 & 8 \\
0 & 8 \\
n & 0 \\
11 & 1\end{array}$ & $\begin{array}{ll}\infty & \infty \\
\sim & 0 \\
\sim & 0 \\
11 & 0\end{array}$ & 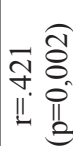 \\
\hline$\prod_{-1}^{0}$ & 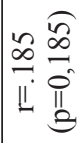 & 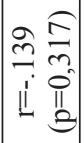 & $\stackrel{1}{\|}$ & 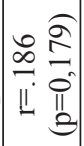 & 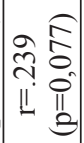 & $\begin{array}{cc} & 0 \\
\infty & 0 \\
= & 0 \\
1 & 0 \\
1 & 0 \\
-1 & 0\end{array}$ & 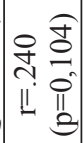 & 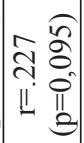 & 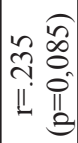 & 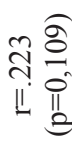 & 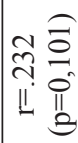 & 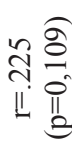 & 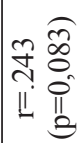 & 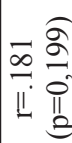 & 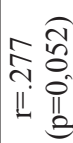 \\
\hline 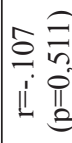 & 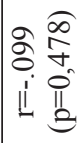 & 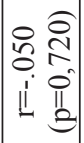 & 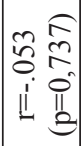 & 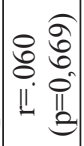 & 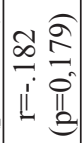 & 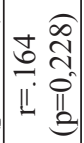 & $\mid \begin{array}{ll}n & 0 \\
n & 0 \\
0 & 0 \\
1 & 0 \\
1 & 0\end{array}$ & 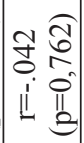 & 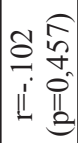 & $\begin{array}{l}0 \\
\hat{n} \\
0 \\
0 \\
1 \\
\| \\
\|\end{array}$ & $\begin{array}{ll} & 0 \\
2 & n \\
0 & 0 \\
0 & 0 \\
11 & 11\end{array}$ & 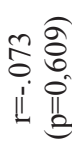 & 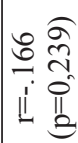 & $\begin{array}{ll}0 & 0 \\
0 & \infty \\
0 & 0 \\
1 & 0 \\
1 & 0\end{array}$ & $\begin{array}{l}8 \\
\stackrel{0}{0} \\
\underline{11}\end{array}$ \\
\hline 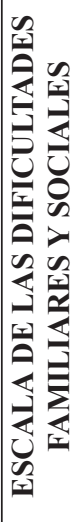 & 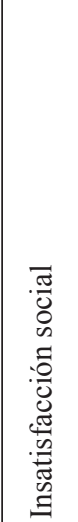 & 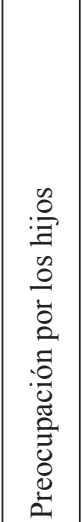 & 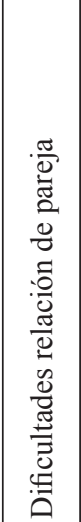 & 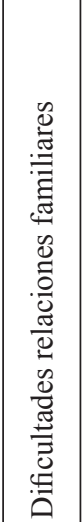 & 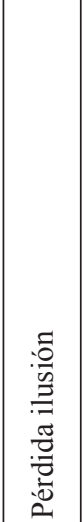 & 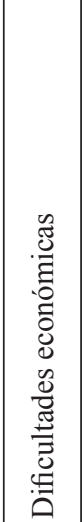 & 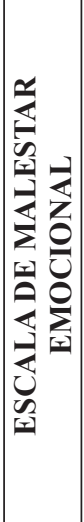 & 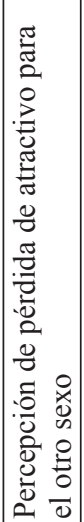 & $\Xi$ & 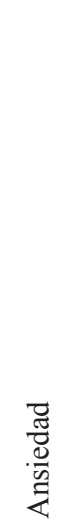 & 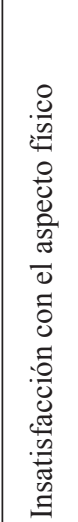 & $\stackrel{\frac{O}{0}}{\stackrel{0}{z}}$ & 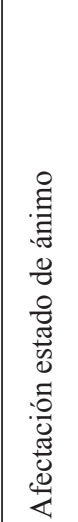 & 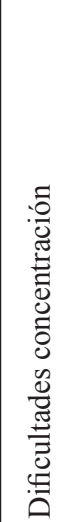 & 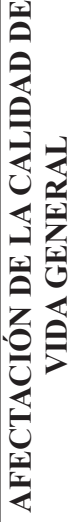 \\
\hline
\end{tabular}




\section{Discusión}

No se observan diferencias significativas en las variables sociodemográficas consideradas, entre las pacientes metastásicas y no metastásicas, por lo que podemos considerar que ambas muestras son comparables. En cuanto a los aspectos médicos registrados, únicamente se observan diferencias significativas en relación a la cirugía. En el momento de la entrevista, todas las pacientes han sido intervenidas quirúrgicamente, excepto 4 pacientes, con metástasis en el momento del diagnóstico (estadio IV de la enfermedad).

La edad media de las mujeres con metástasis es de 60,8 años, en cambio en el grupo sin metástasis, media de edad es inferior (55,7años). Estas diferencias, aunque no son significativas, reflejan la realidad de las dos situaciones de enfermedad. Con el paso del tiempo aumenta la probabilidad de metástasis. En el estudio GEICAM $(2015)^{(36)}$ la media de edad de las pacientes diagnosticadas con metástasis es de 65 años y en mayoría son postmenopáusicas (79\%). En las pacientes más jóvenes mayoritariamente la aparición de la metástasis vendría dada por una recidiva de la enfermedad con una media de edad de 56 años, que seguirían en su mayoría estando en la fase postmenopáusica. Las pacientes de la muestra están de baja laboral o sin empleo por motivos de salud el 53\% y el 32\% restante por otros motivos. Ninguna de las pacientes con metástasis trabajaba en el momento de la valoración, y sólo el 13\% de las pacientes sin metástasis continuaban su actividad profesional. Estos datos reflejan las dificultades a las que se enfrentan estas pacientes a nivel profesional: pueden experimentar problemas de discriminación laboral, despidos injustificados, limitaciones de responsabilidades laborales etc. Todo ello puede afectar no sólo a la capacidad económica. Con frecuencia se observan repercusiones familiares y psicológicas, como la disminución de la autoestima ${ }^{(37)}$.

Respecto a los diferentes componentes de la calidad de vida, observamos que los síntomas son parecidos en los dos grupos, aunque las pacientes con metástasis obtienen puntuaciones más altas en todos los ítems, especialmente en náuseas, pérdida de apetito y fatiga. Las pacientes con metástasis también presentan más dificultades en los hábitos cotidianos. Las diferencias respecto al grupo sin metástasis son significativas respecto al total de la escala y respecto a los ítems referidos a la dificultad para realizar actividades físicas y las tareas de casa. Este peor estado físico y funcional es compatible con el mayor avance de la enfermedad y las posibles secuelas y efectos secundarios de la quimioterapia y la radioterapia. El dolor suele aparecer en las zonas donde se han desarrollado las metástasis, pero también es muy importante la fatiga y el malestar general ${ }^{(38)}$. En cambio, las puntuaciones en la subescala "dificultades familiares y sociales" son parecidas en ambos grupos. Sorprendentemente, las pacientes sin metástasis perciben más dificultades a nivel social, aunque no de forma significativa. Ello podría ser debido a un proceso temporal de adaptación al entorno de la paciente (las pacientes metastásicas conviven con la enfermedad desde hace más tiempo). En lo que se refiere a la subescala de malestar emocional, tal como era de esperar, las pacientes con metástasis muestran mayores puntuaciones, aunque las diferencias no llegan a ser significativas, excepto en "insatisfacción con el aspecto físico". Las pacientes con metástasis tienen puntuaciones significativamente más elevadas, probablemente debido a un conjunto de factores relacionados con los tratamientos de larga duración, como la quimioterapia y la hormonoterapia, por sus efectos en el estado físico como la fatiga, y la alteración de la imagen corporal. 
Así pues, en síntesis, la hipótesis 1 según la cual la calidad de vida sería más favorable en el grupo de pacientes sin metástasis, sólo se cumple a nivel de dificultades en los hábitos cotidianos e insatisfacción en el aspecto físico. En los demás aspectos, incluyendo la valoración general de la calidad de vida, las diferencias no son significativas. Estos resultados difieren de otros anteriores, en los que se observan diferencias a nivel físico y emocional, como los de Butlet et al. $(2003)^{(27)}$, mientras que son coherentes los obtenidos en un estudio anterior, donde se observó que la mitad de las pacientes con metástasis de larga evolución opinaban que la enfermedad había aportado un valor positivo a sus vidas ${ }^{(39)}$.

Respecto a las respuestas de afrontamiento, no se observan diferencias significativas entre los grupos: todas las pacientes pueden utilizar estrategias de afrontamiento similares (hipótesis 2). La estrategia más utilizada en pacientes con metástasis es la "Evitación Cognitiva" y en pacientes sin metástasis el "Fatalismo", pero sin que se observen diferencias significativas. Las estrategias pueden ser usadas por todas las pacientes, independientemente de que presenten o no metástasis, coincidiendo con Font y Cardoso ${ }^{(25)}$. En cambio, en el estudio de Grassi $^{(40)}$ la estrategia más utilizada era la de preocupación ansiosa, mientras que la evitación cognitiva era la menos usada.

Los estilos de afrontamiento se diferenciaron en parte respecto a su relación con la calidad de vida (hipótesis 3). En la presente investigación las respuestas Preocupación Ansiosa y Desesperanza se muestran vinculadas significativamente con una disminución de la calidad de vida general. Por otra parte, las reacciones de afrontamiento pasivas como la Desesperanza, la Preocupación Ansiosa, la Evitación Cognitiva y el Fatalismo, se relacionan también con mayor afectación de la calidad de vida. Estos resultados son coherentes con los obtenidos por otros autores ${ }^{(21,41,42,43)}$ : las pacientes con un afrontamiento más activo tenían mejor calidad de vida, especialmente a nivel psicosocial, mientras que las pacientes más pesimistas suelen presentar peor calidad de vida ${ }^{(29)}$. La estrategia de afrontamiento "evitación" también se mostró como factor predictor de peor calidad de vida, coincidiendo con estudios anteriores ${ }^{(23,29)}$. Respecto a la estrategia "espíritu de lucha", en general correlacionó con menor afectación de la calidad de vida (en 15 de los 26 ítems específicos). Pero, contrariamente a lo esperado, se observó una mayor afectación de los hábitos cotidianos y más dificultades en el cuidado personal. Es posible que la presencia de dificultades en estas áreas active el espíritu de lucha, lo que a su vez facilitaría una disminución de las dificultades familiares y sociales, y un mejor estado emocional.

Entendemos que la principal limitación del presente estudio para conocer de manera precisa la relación entre las diferentes respuestas en las dos condiciones de salud analizadas puede ser el tamaño de muestra, pero los dos grupos de pacientes comparten muchas características en común, lo que facilita su comparación. La información obtenida puede ser interesante para futuras investigaciones sobre las diferentes respuestas al cáncer y su relación con la calidad de vida en pacientes con y sin metástasis, y será ampliada una vez finalice el estudio del que forma parte, con el objetivo último de contribuir a una mejor comprensión de las respuestas que facilitan la adaptación exitosa al cáncer de mama en situación avanzada. 


\section{Conclusión}

Pese a que la calidad de vida es más favorable a nivel de hábitos cotidianos y satisfacción con el aspecto físico en el grupo de pacientes sin metástasis, la calidad de vida a nivel familiar, social y emocional es parecida, y ambos grupos de pacientes utilizan formas similares de afrontamiento.

\section{Referencias bibliográficas}

1. Organización Mundial de la Salud OMS. Cáncer de mama: prevención y control. [Acceso el 1 de febrero de 2020]. Disponible en: https://www.who.int/topics/cancer/breastcancer/ es/index1.html. 1.7.2019.

2. Font A. Cáncer y calidad de vida. Anuario de Psicología 1994; 61: 41-50.

3. Calvo D, Moreno M, Alonso B, Álvarez M, Lana A, Llaneza A. Calidad de vida relacionada con la salud y peso corporal en mujeres supervivientes al cáncer de mama. Rev Senol Patol Mamar 2018; 31;31-5. doi: 10.1016/J.SENOL.2018.03.002

4. Qaseem A, Lin J, Mustafa R, Horwitch C, Wilt T. Screening for breast cancer in averagerisk women: A guidance statement from the American College of Physicians. Ann Intern Med 2018; 170: 547-60. doi: 10.7326/M18-2147

5. Folkman S, Lazarus RS. An analysis of coping in a middle-aged community sample. J Health Soc Behav 1980; 21:219-39.

6. Roussi P, Krikeli V, Hatzidimitriou C, Koutri I. Patterns of coping, flexibility in coping and psychological distress in women diagnosed with breast cancer. Cognit Ther Res 2007; 31:97-109.

7. Nipp R, El-Jawahri A, Fishbein J, Eusebio J, Stagl J, Gallagher E, et al. The relationship between coping strategies, quality of life, and mood in patients with incurable cancer. Cancer 2016; 122: 2110-6. doi: 10.1002/cncr.30025

8. Yoo G, Sudhakar A, Le M, Levine E. Exploring Coping Strategies Among Young Asian American Women Breast Cancer Survivors. J Canc Educ 2017;32: 43-50. doi: 10.1007/ s13187-015-0917-x

9. Watson M, Greer S. Personality and Coping. En Holland J, editor. Psycho-Oncology. Nueva York: Oxford University Press, 1998.p.91-98.

10. De Haro M, Gallardo L, Martínez L, Camacho N, Velázquez J, Paredes E. Factores relacionados con las diferentes estrategias de afrontamiento al cáncer de mama en pacientes de recién diagnóstico. Psicooncología 2014; 11: 87-99. doi: 10.5209/rev_ PSIC.2014.v11.n1.44919.

11. You J, Wang C, Rodriguez L, Wang X, Lu Q. Personality, coping strategies and emotional adjustment among Chinese cancer patients of different ages. Eur J Cancer Care 2018; 27, 1-9. doi: $10.1111 /$ ecc. 12781.

12. Haber, S. Cáncer de mama: Manual de tratamiento psicológico. España: Paidós, 2000.

13. Martín D, Zanier J, García F. Afrontamiento y calidad de vida. Un estudio de pacientes con cáncer. Psico-USF 2003, 8,175-82.

14. Body G, Suzanne I, Senon J, Brunault P, Voyer M, Huguet G, et al. Major depressive disorder, personality disorders, and coping strategies are independent risk factors for lower quality of life in non-metastatic breast cancer patients. Psychooncology 2015; 520,: 513-20. doi: 10.1002/pon.3947 
15. Manne S, Glassman M, Du Hamel K. Intrusion, avoidance, and psychological distress among individuals with cancer. Psychosom Med 2000; 63: 658-67.

16. Paek M, Levine B, Avis N. Longitudinal reciprocal relationships between quality of life and coping strategies among women with breast cancer. Ann Behav Med 2016; 50: 77583. doi: 10.1007/s12160-016-9803-y

17. Dunkel-Schetter C, Feinstein L, Taylor S, Falke R. Patterns of coping with cancer. Health Psychol 1992; 11: 79-87.

18. Mytko J, Knight S, Chastain D, Mumby P, Siston A, Williams S. Coping strategies and psychological distress in cancer patients before autologous Bone Marrow Transplant. J Clin Psychol 1996; 3: 355-66.

19. Classen C, Koopman C, Angell K, Spiegel D. Coping styles associated with psychological adjustment to advanced breast cancer. Health Psychol 1996; 15: 434-7.

20. Hack T, Degner L. Coping responses following breast cancer diagnosis predict psychological adjustment three years later. Psycho-Oncology 2004; 13: 235-47. doi: 10.1002/pon.739

21. Mera P, Ortiz M. La relación del optimismo y las estrategias de afrontamiento con la calidad de vida de mujeres con cáncer de mama. Ter Psicol 2012;30: 69-78. doi: 10.4067/ S0718-48082012000300007

22. Alcalar N, Ozkan S, Kucucuk S, Aslay I, Ozkan M. Association of Coping Style, Cognitive Errors and Cancer-related Variables with Depression in Women Treated for Breast Cancer, Jpn J Clin Oncol 2012; 42: 940-947. doi: 10.1093/jjco/hys119

23. Torrecilla M, Martín L, Riva J. Afrontamiento y calidad de vida en mujeres pacientes oncológicas. Psiencia 2016; 8: 1-16. Doi: 10.5872/psiencia/8.3.21

24. Greer S, Morris T, Pettingale. Psychological response to breast cancer: effect on outcome. Lancet 1979; 2: 785-7.

25. Font A, Cardoso A. Afrontamiento en cáncer de mama: Pensamientos, conductas y reacciones emocionales. Psicooncologia, 2009; 6: 27-42.

26. Kvillemo P, Bränström R. Coping with breast cancer: A meta-analysis. Plos One 2014; 9:112733. doi: 10.1371/journal.pone.0112733

27. Butler L, Koopman C, Cordova M, Garlan R, DiMIceli S, Spiegel D. Psychological distress and pain significantly increase before death in metastatic breast cancer patients. Psychosom Med 2003;65:416-26. doi: 10.1097/01.PSY.0000041472.77692.

28. Hagedoorn M, Sanderman R, Bolks HN, Tuinstra J, Coyne JC. Distress in couples coping with cancer: A meta-analysis and critical review of role and gender effects. Psychol Bull 2008;134:1-30. doi: 10.1037/0033-2909.134.1.1.

29. Wang W, Tu P, Liu T, Yeh D, Hsu W. Mental adjustment at different phases in breast cancer trajectory: re-examination of factor structure of the Mini-MAC and its correlation with distress. Psychooncology, 2013;774, 768-774. doi: 10.1002/pon.3065.

30. Pastells P, Font A. Optimismo disposicional y calidad de vida en mujeres con cancer de mama. Psicooncología 2014; 11: 19-29. doi: 10.5209/rev_PSIC.2014.v11.n1.44914

31. Cheng C, Liu W, Wang G, Lim L, Gao S, Ho S, et al. Cancer-coping profile predicts longterm psychological functions and quality of life in cancer survivors. Support Care Cancer 2019;27: 933-41. doi. 10.1007/s00520-018-4382-z

32. Costa-Requena G, Gil F. The mental adjustment to cancer scale: a psychometric analysis in Spanish cancer patients. Psychooncology, 2009; 18: 984-91.

33. Font A. Estrategias de afrontamiento, adaptación y calidad de vida Psicología. Psicología española en la Europa de los 90: ciencia y profesión, Valencia, 15 a 20 de abril de 1990; 7: $90-6$ 
34. Font A, Rodríguez E, Buscemi, V. Fatiga, expectativas y calidad de vida en Cáncer. Psicooncología 2004;1: 45-56.

35. Espantoso R, Fernández C, Padierna C, Amigo I, Villoria E, Gracia J, et al. Calidad de vida en pacientes oncológicos un año después de finalizado el tratamiento. Psicooncología 2007; 4: 43-57.

36. GEICAM. Cáncer de mama metastásico. EMC - Ginecología-Obstetricia 2015; (Vol. 51). doi: 10.1016/S1283-081X(15)70034-2

37. Sebastián J, Manos D, Bueno J, Mateos N. Imagen corporal y autoestima en mujeres con cáncer de mama participantes en un programa de intervención psicosocial. Clin Salud 2007; 18: 137-61.

38. Vivar C. Impacto psicosocial del cáncer de mama en la etapa de larga supervivencia: propuesta de un plan de cuidados integral para supervivientes. Aten Primaria 2012; 44: 288-92. doi: 10.1016/j.aprim.2011.07.011

39. Falo C, Villar H, Rodríguez E, Mena E, Font A. Calidad de vida en cáncer de mama metastásico de larga evolución: la opinión de las pacientes. Psicooncología 2014; 11 : 313-31. Doi: 10.5209/rev_PSIC.2014.v11.n2-3.47391.

40. Grassi L, Buda P, Cavana L, Annunziata M, Torta R, Varetto A. Styles of coping with cancer: the Italian version of the Mini-Mental adjustment to cancer (MINI-MAC) Scale. Psychooncology 2005;14: 115-24.

41. Galindo O. Escala de afrontamiento al cáncer en pacientes oncológicos en población mexicana. Psicooncología 2019;16: 61-72. Doi:10.5209/PSIC.63648

42. Niu L, Liang Y, Niu M. Factors influencing fear of cancer recurrence in patients with breast cancer: Evidence from a survey in Yancheng, China. J Obstet Gynaecol Res 2019; 45:1319-27. doi: 10.1111/jog.1397

43. Taghian N, Miller C, Jammallo L, O’Toole J, Skyolny M. Lymphedema following breast cancer treatment and impact on quality of life: a review. Psychol Bull 2014;134:1-30. doi: 10.1037/0033-2909.134.1.1. 